\title{
Investigación en Atención Primaria en Castilla-La Mancha
}

\author{
Antonio Segura Fragoso ${ }^{a}$
}

\begin{abstract}
a Jefe de Servicio de Investigación.

Instituto de Ciencias de la Salud.
\end{abstract}

\section{Correspondencia:}

Antonio Segura Fragoso.

Instituto de Ciencias de la

Salud, Crta. de Extremadura

$\mathrm{Km} \mathrm{114,1.}$

45600-Talavera de la Reina.

Telf 925839230 ,

e-mail: asegura@jccm.es.

Recibido el 20 de diciembre de 2007.

\section{Aceptado para su}

publicación el 10 de enero de 2008.

\section{RESUMEN}

La producción científica de España en Atención Primaria aumenta a un ritmo notable en los últimos años, ocupando el cuarto lugar entre los países que muestran una mayor proporción de producción reciente. En Castilla-La Mancha la producción científica de Atención Primaria también ocupa el cuarto lugar entre las CCAA en cuanto a producción por millón de habitantes, siendo un lugar destacado dentro de la producción científica española. La evolución temporal de los indicadores de impacto y citas recibidas es positiva mostrando tendencias a mejorar en los años recientes. La evolución del factor de impacto medio de las revistas en las que se publica va mejorando con los años. Esto significa que los profesionales de Atención Primaria en Castilla-La Mancha cada vez publican en revistas de mejor calidad.

Palabras clave. Investigación. Atención Primaria de Salud.

\section{ABSTRACT}

\section{Primary care research in Castilla-La Mancha.}

In Spain scientific production in primary care has increased at a remarkable rate in the last few years. Spain now occupies fourth place amongst the countries with the highest rate of recent production. Castilla-La Mancha also occupies fourth place amongst the Spanish autonomous communities in scientific production in Primary Care, as regards production per million inhabitants. Temporal progression of the impact factors and citations is positive and has shown an increase in recent years. The mean impact factor of the journals in which primary care articles are published is increasing every year. This means that Primary Care professionals in Castilla-La Mancha are publishing in higher quality journals more frequently.

Key Words. Research. Primary Health Care.

\section{INTRODUCCIÓN}

La mayor parte de los profesionales tenemos la sensación de que la cantidad y la calidad de la investigación en Atención Primaria que se realiza en Castilla-La Mancha es insuficiente y con frecuencia nos preguntamos qué se podría hacer para mejorar esta situación. En realidad no disponemos de datos objetivos que nos permitan cuantificar el nivel en que se encuentra nuestra región en comparación con otras comunidades autónomas, ni tampoco el lugar en que se encuentra España en comparación con otros países de nuestro entorno en investigación en Atención Primaria. Sí sabemos que, según datos del Instituto Nacional de Estadística, en I+D+i España se encuentra, en general, a la cola de los países desarrollados en el porcentaje del PIB que dedica a la investigación y desarrollo. También sabemos que, dentro de España, Castilla-La Mancha se encuentra a la cola de las comunidades autónomas en inversión en I+D+i. 
Pero, ¿ocurre lo mismo en Atención Primaria? ¿Es similar la situación de nuestra región en investigación en Atención Primaria que en I+D+i en general?

Para intentar situar la posición de la investigación en Atención Primaria de Castilla-La Mancha en el contexto del resto de las comunidades autónomas y de Europa, así como describir sus propias peculiaridades internas, se proponen tres preguntas a responder:

1. ¿Qué lugar ocupa España en relación a los países europeos en investigación en Atención Primaria?

2. ¿Qué lugar ocupa Castilla-la Mancha en relación al resto de las comunidades autónomas españolas?

3. ¿Cómo es y cómo evoluciona en los últimos años la investigación en Atención Primaria en nuestra región?

La respuesta a estas preguntas es conveniente para poder valorar el lugar en que nos encontramos. Independientemente de que nuestra situación sea mejor o peor, siempre se podrá y se deberá mejorar. Por ello en la última parte de este artículo se abordará una cuarta cuestión:

4. ¿Qué estrategias serían adecuadas para impulsar la investigación en Atención Primaria en Castilla-La Mancha?

El método que se ha utilizado para medir la cantidad de investigación realizada es la medida de la producción científica a través de los documentos que pueden ser recuperados de las bases de datos bibliográficas. Por tanto, es una medida de resultados de investigación. Se considera que toda investigación, tanto sea la realizada a través de un proyecto formal financiado por una agencia con evaluación externa como la producida por una investigación más espontánea realizada en la propia consulta (pero no por ello menos interesante), debe acabar en una publicación, ya que de lo contrario se elimina uno de los actos esenciales de la producción de conocimiento que es la difusión

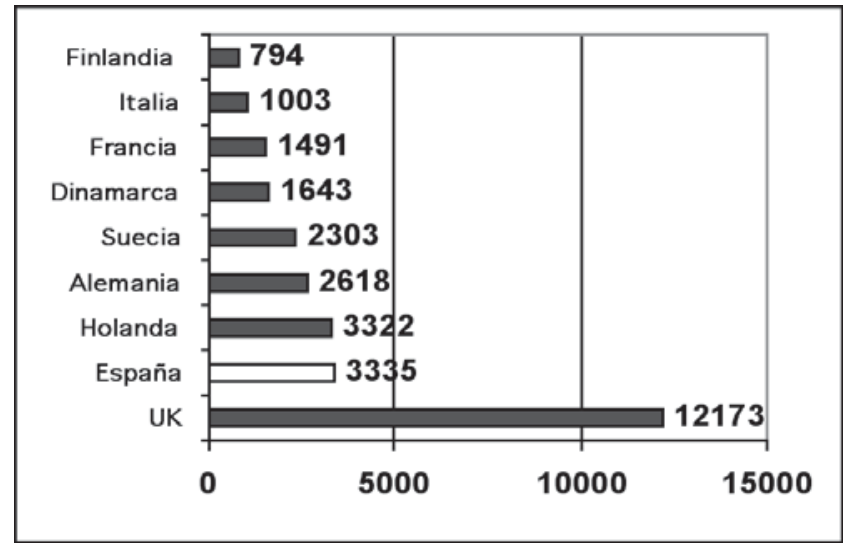

Figura 1. PubMed. Documentos publicados de Atención Primaria.

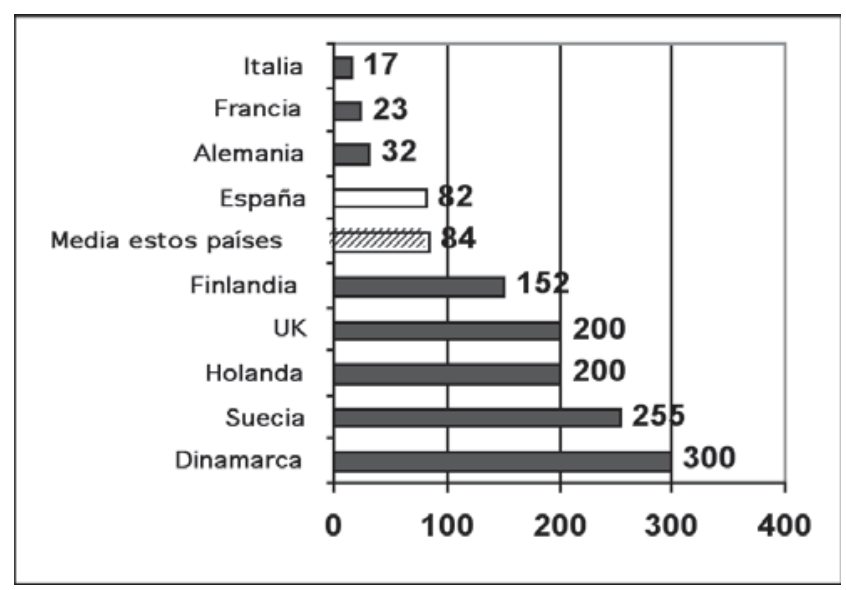

Figura 2. PubMed. Documentos publicados de Atención Primaria por millón de habitantes.

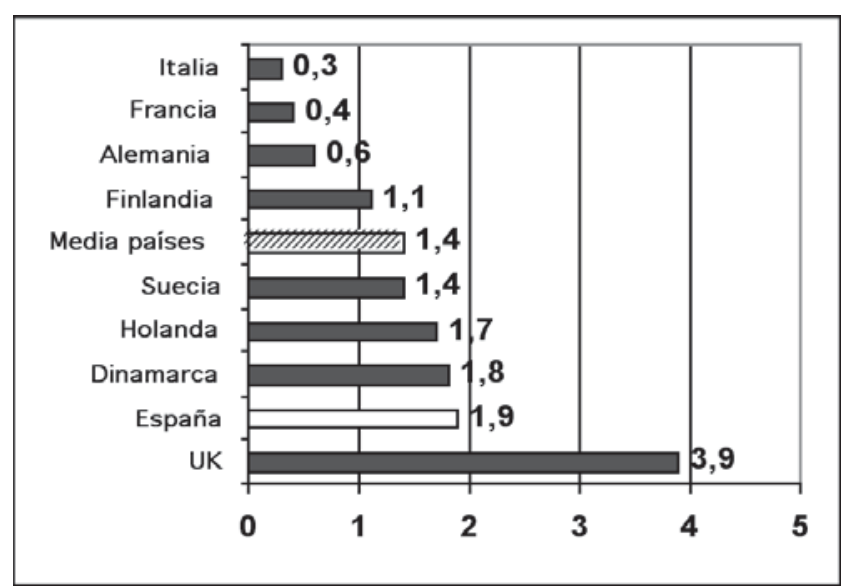

Figura 3. PubMed. \% Documentos de Atención Primaria sobre el total de documentos del país.

de la investigación, con la consiguiente posibilidad de que los métodos y resultados sean conocidos y criticados por la comunidad científica y profesional.

En este trabajo se ha utilizado las base de datos PubMed, la base de datos del Indice Médico Español y la base de datos de la WOK (Science Citation Index).

\section{¿QUÉ LUGAR OCUPA ESPAÑA EN RELACIÓN A LOS PAÍSES EUROPEOS EN INVESTIGACIÓN EN ATENCIÓN PRIMARIA?}

Para responder a esta pregunta comparativa entre países se ha realizado una búsqueda bibliográfica en la base de datos PubMed, que es accesible a todos los países estudiados. Se han buscado documentos publicados "que traten sobre Atención Primaria" en sentido amplio. En concreto se han buscado los documentos que contengan como descriptores $\mathrm{MeSH}$ alguno de los siguientes: \{primary health care\} OR \{Family Practice\} OR \{Physicians, Family\} OR \{General Practice\}. Además se han incluido aquéllos que 


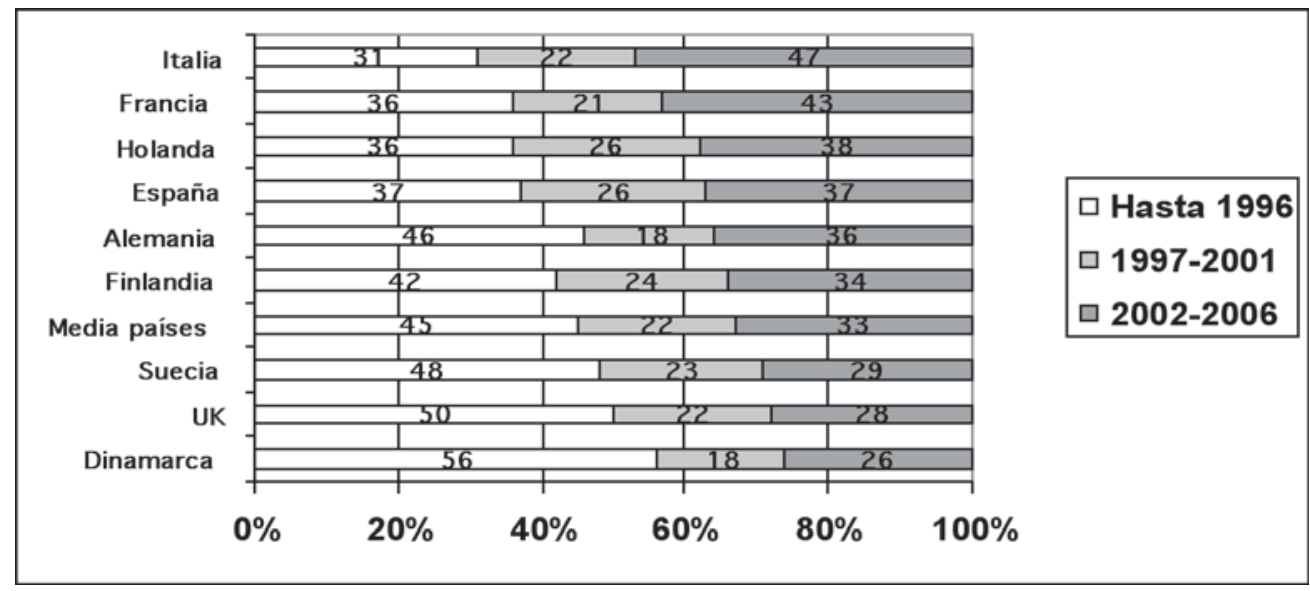

Figura 4. PubMed. \% Documentos de Atención Primaria en diferentes periodos.

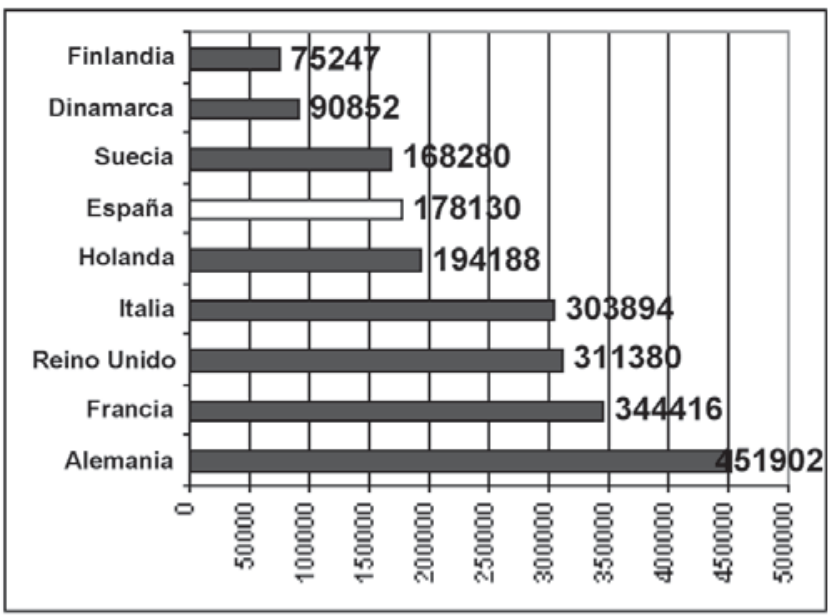

Figura 5. PubMed. Total documentos publicados.

contengan en el título o en el resumen alguno de los siguientes términos: \{primary health care\} OR \{Family Practice OR \{Physicians, Family\} OR \{general practice\} OR \{family medicine\} OR \{health center\} OR \{primary care\}. Los países que se han comparado con España son Alemania, Francia, Reino Unido, Italia, Holanda, Suecia, Dinamarca y Finlandia, es decir, los países europeos de nuestro entorno.

El número de documentos sobre Atención Primaria localizados en PubMed pertenecientes a este conjunto de países ha sido de 28.682. En la figura 1 se aprecia el ranking de países por mayor número de documentos publicados sobre Atención Primaria. España ocupa el segundo lugar, solo por detrás del Reino Unido, que destaca sobre todos los demás.

Debido al diferente tamaño de la población y por tanto del número de profesionales de Atención Primaria que publican, es necesario a efectos comparativos utilizar un indicador relativo. En la figura 2 se ofrece el

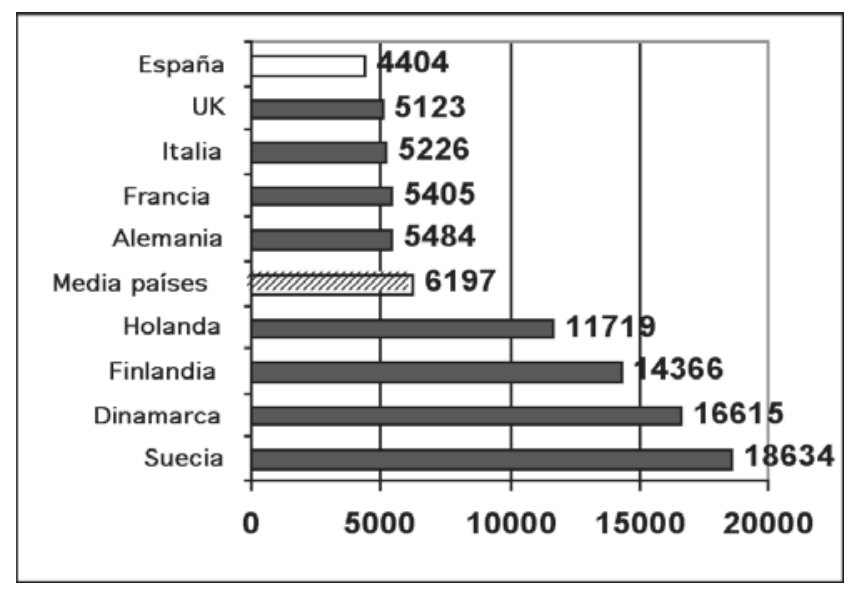

Figura 6. PubMed. Total documentos publicados por millón de habitantes.

ranking de producción científica en Atención Primaria por millón de habitantes. España se sitúa prácticamente en la media de producción científica del grupo de países estudiados, muy por delante de Alemania, Francia o Italia, aunque bastante alejada de los países nórdicos y el Reino Unido, cuyo colectivo de profesionales de Atención Primaria tienen tradicionalmente una elevada producción científica.

Un aspecto interesante a comparar es qué peso tiene la investigación específica en Atención Primaria respecto a la producción total del país en Biomedicina y Ciencias de la Salud. En la figura 3 se observa que España ocupa el segundo lugar con un 1,9\%, bastante por encima de la media del grupo de países, que se sitúa en el 1,4\%.

La producción científica en Atención Primaria en España muestra una tendencia claramente creciente entre 1997 y 2006, de forma similar a países como Holanda. Otros países muestran un perfil de menor 


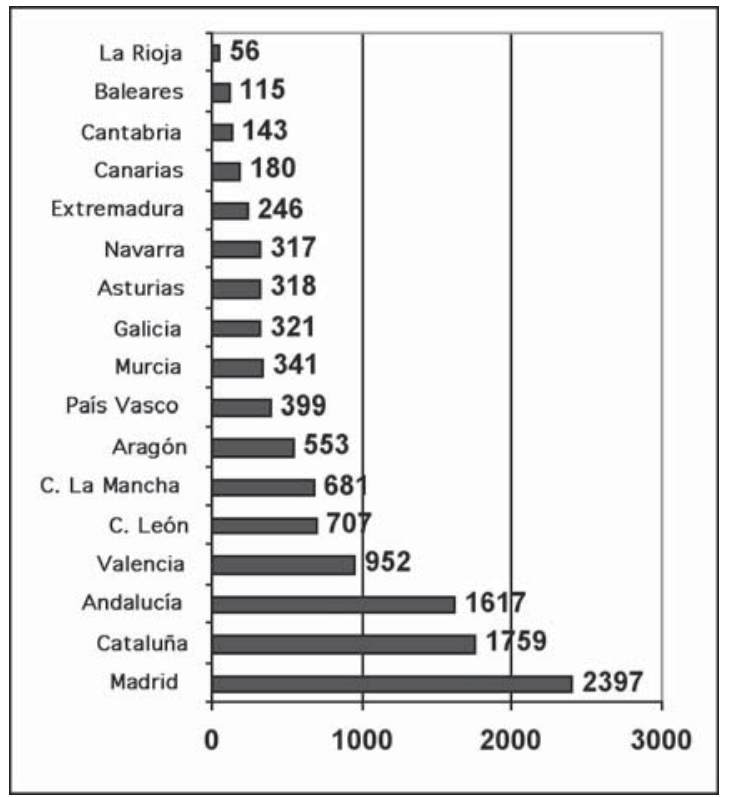

Figura 7. Producción científica hecha en atención primaria. Número total de documentos.

crecimiento e incluso de cierta estabilización como Suecia, o Dinamarca. Esta misma información se corrobora en la figura 4, en la que se aprecia cómo España ha producido en Atención Primaria entre 2002 y 2006 tanto como en toda su historia hasta 1996, ocupando el cuarto lugar entre los países que tienen mayor proporción de publicaciones recientes.

Como conclusiones generales en este apartado se podrían señalar las siguientes:

- España ocupa un lugar destacado en producción absoluta en Atención Primaria y un lugar intermedio en producción relativa al número de habitantes, en comparación con el grupo de países estudiados.

- La producción científica de España en Atención Primaria aumenta a un ritmo notable en los últimos años ocupando el cuarto lugar entre los países que muestran una mayor proporción de producción reciente.

Pero, ¿este nivel observado en la producción científica española en Atención Primaria se corresponde con el nivel de su producción científica en Biomedicina y Ciencias de la Salud? ¿Es mejor? ¿Es peor? Con objeto de comparar el potencial de los países estudiados respecto a su producción científica en Biomedicina y Ciencias de la salud en su conjunto, se ha localizado la totalidad de los documentos publicados en PubMed por cada uno de los países. En las figuras 5 y 6 se aprecia cómo la posición de España en la producción científica en biomedicina y ciencias de la salud es notablemente más retrasada que la observada en Atención Primaria (figuras 1 y 2), ocupando el

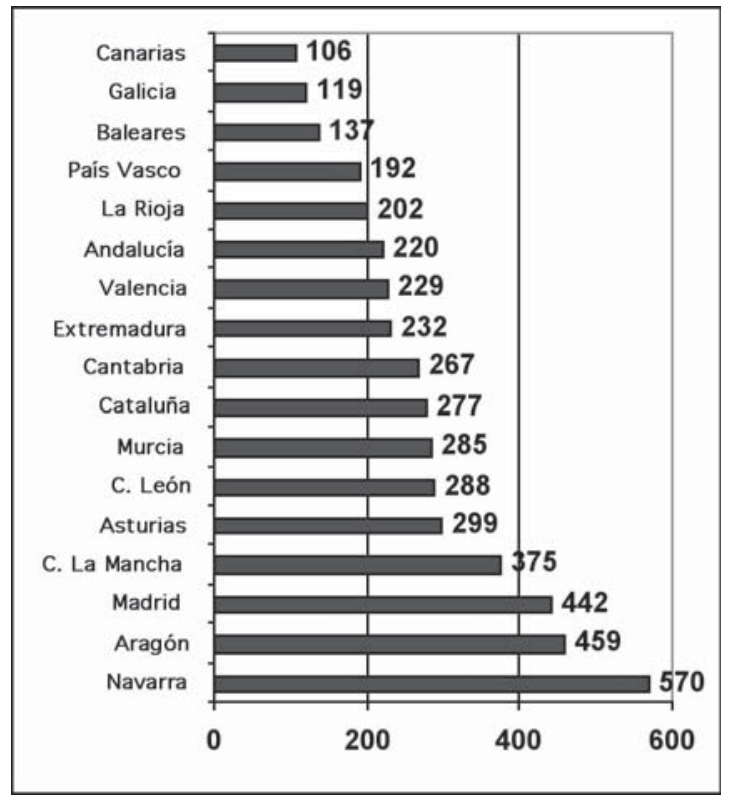

Figura 8. Producción científica hecha en atención primaria. Número de documentos por millón de habitantes.

sexto lugar en producción absoluta y el último lugar en producción relativa al número de habitantes. Esto significa que la posición de España en Atención Primaria es claramente mejor que la posición en biomedicina y ciencias de la salud.

\section{¿QUÉ LUGAR OCUPA CASTILLA-LA MANCHA EN RELACIÓN AL RESTO DE LAS COMUNIDADES AUTÓNOMAS ESPAÑOLAS?}

La metodología para responder a esta pregunta cambia sustancialmente con respecto a la anterior en dos aspectos significativos. El primero es que para analizar la producción científica dentro de España y poder comparar las comunidades autónomas entre sí se hace necesario incluir las publicaciones realizadas en revistas editadas en España que no estén incluidas en PubMed, ya que el $80 \%$ de la producción científica en Atención Primaria se realiza fuera de PubMed. Por ello se ha utilizado también la base de datos del IME (Indice Médico Español). El segundo cambio es que la estrategia de búsqueda ha ido encaminada a localizar documentos "hechos en Atención Primaria" en lugar de "que traten sobre Atención Primaria". Para ello se han buscado en IME y en PubMed documentos que contengan en el Lugar de Trabajo de los autores algún indicativo de ser un centro de atención primaria en el sentido más amplio (centro de salud, EAP, gerencias de atención primaria, unidades de medicina familiar y comunitaria, etc.) y además se han incluido en la búsqueda documentos que contengan alguno de estos indicativos en el título. Los documentos recuperados 


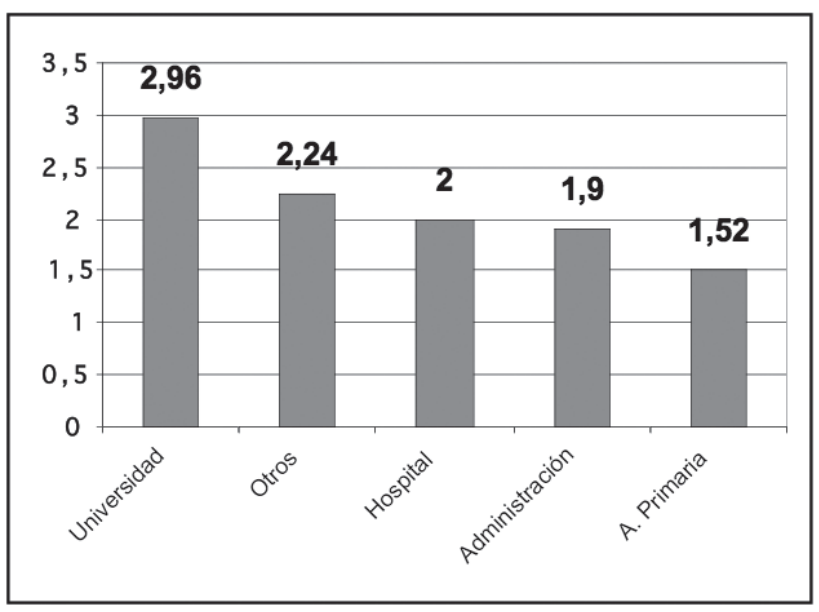

Figura 9. Factor de Impacto medio por tipo de centro. Documentos citables publicados en revistas ISI-JCR. Castilla-La Mancha, 1971-2005.

han sido examinados de forma manual para comprobar la participación de algún centro relacionado con la Atención Primaria.

El número de documentos recuperados ha sido de 15.295. Se ha podido identificar la participación de al menos un centro relacionado con la Atención Primaria en 9.199 de ellos. Cada uno de estos artículos ha sido revisado manualmente y asignado el tipo de centro y provincia del/los centros de Atención Primaria participantes. El número de centros participantes con el que se han elaborado los gráficos ha sido de 11.034

Castilla-La Mancha ocupa el sexto lugar entre las CCAA españolas en número absoluto de documentos (figura 7). Debido al diferente tamaño de las mismas conviene utilizar una medida relativa. En la figura 8 se aprecia que la Atención Primaria de nuestra región ocupa el cuarto lugar entre las CCAA en producción por millón de habitantes, al igual que sucede si el cálculo se realiza por mil médicos de EAP o por mil profesionales de EAP, por detrás de Navarra, Aragón y Madrid. La conclusión es que la Atención Primaria de Castilla-La Mancha ocupa un lugar destacado dentro de la producción científica española.

\section{¿CÓMO ES Y CÓMO EVOLUCIONA EN LOS ÚL- TIMOS AÑOS LA INVESTIGACIÓN EN ATENCIÓN PRIMARIA EN NUESTRA REGIÓN?}

Para responder a esta pregunta se ha utilizado la base de datos descrita en mayor detalle en la reciente publicación1 sobre la Producción científica de CastillaLa Mancha en Biomedicina y Ciencias de la Salud, 1973-2003, pero actualizada hasta el año 2005. En

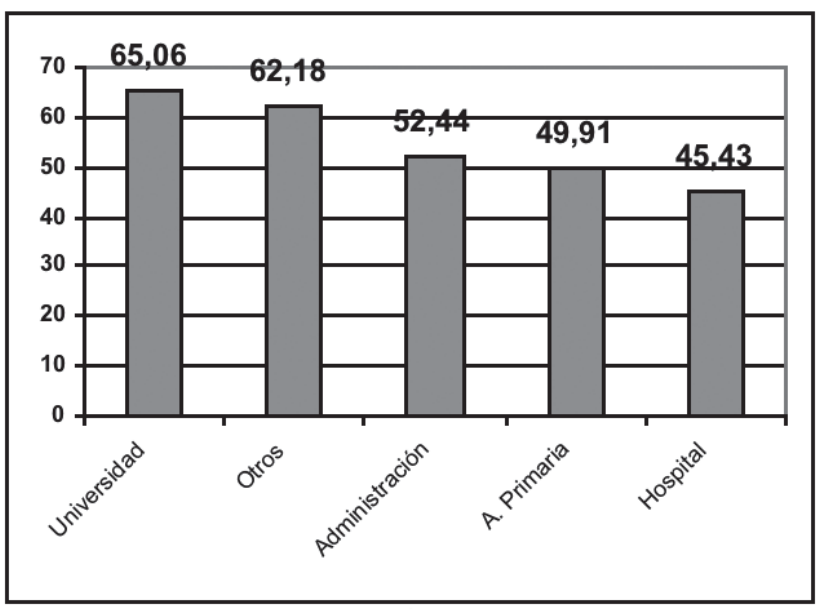

Figura 10. Percentil medio del Factor de Impacto por tipo de centro. Documentos citables publicados en revistas ISI-JCR. Castilla-La Mancha, 1971-2005.

resumen, se trata de la recopilación a partir de las bases de datos ya mencionadas WOK, PubMed e IME de todos los documentos sobre Biomedicina y Ciencias de la Salud en los que se pueda reconocer la participación de un autor perteneciente a un centro de Castilla-La Mancha. El resultado es la recopilación de 4.796 documentos de Castilla-La Mancha en los cuales han participado centros de nuestra región en 6.419 ocasiones entre 1971 y 2005. De ellos 818 son centros de Atención Primaria. Esta información permite describir con cierto detalle algunas características de la investigación en Atención Primaria.

A continuación se presentan algunos de los resultados más sobresalientes:

- La producción científica de Atención Primaria supone el $13 \%$ en número total de documentos. El $83 \%$ de la misma está publicada en revistas españolas no contenidas en el ISI-JCR, por tanto sin factor de impacto internacional.

- Al estudiar la evolución temporal de las publicaciones de Castilla-La Mancha según los diferentes ámbitos, puede comprobarse que la producción total de la región es claramente creciente, así como la de los hospitales y la universidad. Sin embargo, la producción de Atención Primaria ha sufrido un cierto retroceso en los años 2004 y 2005. Hasta 2003, presentaba una tendencia creciente que no se ha visto confirmada.

- El tipo de investigación que se realiza en Atención Primaria se diferencia claramente de la realizada en los hospitales, la universidad y la propia administración. Su principal característica sería una mayor diversidad y una menor especialización, abarcando la investigación clínica, epidemiológica y de servicios de salud. 


\begin{tabular}{|l|c|}
\hline Revista de Publicación & No \\
\hline Atención Primaria & 178 \\
Semergen & 119 \\
Salud Rural & 66 \\
Centro de Salud & 59 \\
Medicina Clínica & 46 \\
Medifam. Revista de Medicina Familiar y Comunitaria & 31 \\
Journal of Hypertension & 24 \\
SEMER & 21 \\
Medicina General & 17 \\
Revista Española de Salud Pública & 15 \\
Medicina Integral & 14 \\
Revista Clínica Española & 11 \\
Revista Española de Geriatría y Gerontología & 11 \\
Hipertension & 10 \\
Revista de Sanidad e Higiene Pública & 10 \\
\hline
\end{tabular}

Tabla 1. Revistas con mayor número de artículos publicados.

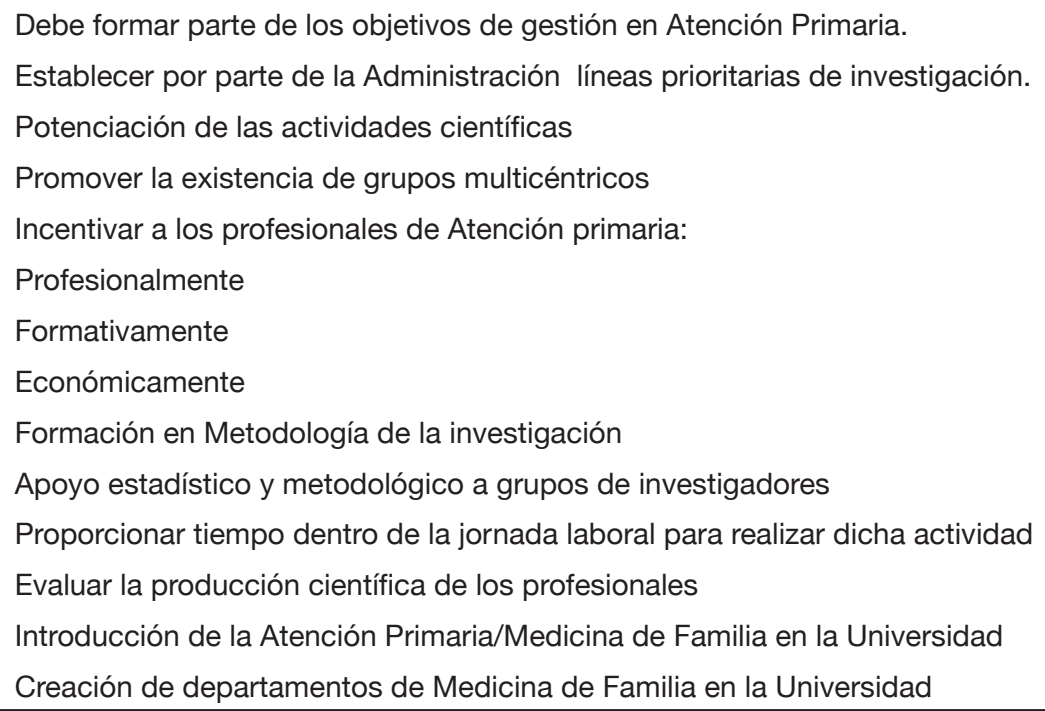

Tabla 2. Condiciones para mejorar la investigación en Atención Primaria.

- La participación en estudios multicéntricos es beneficiosa porque aumenta la calidad e interés de la investigación. Aproximadamente el $50 \%$ de los estudios en que participa Atención Primaria son multicéntricos, por encima de los hospitales que solo lo hacen en un $37 \%$, pero con menos frecuencia que otras instituciones. El $40 \%$ de las colaboraciones multicéntricas de Atención Primaria se realiza con centros de la propia comunidad autónoma.

- Atención Primaria ostenta el menor Factor de Impacto medio entre las instituciones de la región (figu- ra 9). Esto es debido en gran medida al área científica de las revistas en que realiza sus publicaciones, que se ve penalizada al compararla con otras áreas científicas (especialidades clínicas y sobre todo disciplinas básicas) que tienen mayores factores de impacto. Para evitar este problema y permitir que diferentes disciplinas científicas puedan ser comparadas se utilizan los percentiles del factor de impacto (figura 10) observándose cómo la posición de Atención Primaria mejora, mostrando un nivel de calidad muy aceptable, superior a los hospitales. Atención Primaria pu- 
blica en revistas cuyo factor de impacto se encuentra alrededor del percentil 50 en promedio.

Otro aspecto interesante a estudiar es la repercusión que han tenido en la comunidad científica los artículos publicados. Esto se mide habitualmente por medio del número de citas recibidas. Los artículos de Atención Primaria han recibido en promedio algo más de cuatro citas cada uno, número menor que el de las otras instituciones de la región. Sin embargo, cuando se analiza el porcentaje de artículos que no han recibido ninguna cita (porque se supone que no han despertado suficiente interés entre la comunidad profesional y científica) Atención Primaria ocupa un lugar intermedio entre las instituciones de la región con un $25 \%$ de artículos nunca citados, posición claramente mejor que la de las publicaciones de los hospitales cuya tasa de no citación asciende al 35\%. Esto significaría que la producción de Primaria tiene un nivel medio más homogéneo, mientras que la de los hospitales tendría mayor dispersión con artículos de gran interés y otros de escaso interés.

La evolución temporal de los indicadores de impacto y citas recibidas es positiva mostrando tendencias a mejorar en los años recientes. La evolución del factor de impacto medio de las revistas en que se publica va mejorando con los años. Esto significa que los profesionales de Atención Primaria cada vez publican en revistas de mejor calidad.

Para finalizar esta descripción de la producción científica de Atención Primaria se examinarán algunos datos comparativos entre las áreas de salud de Castilla-La Mancha. En cuanto al número absoluto de documentos y el \% de la producción científica de Atención Primaria que aporta cada una de las áreas sanitarias, destacan el área de Toledo y la de Guadalajara. Debido a la diferente población de las áreas, al compararlas según el $\mathrm{n}^{\circ}$ de documentos por 100.000 habitantes permanecen en primer lugar Guadalajara y Toledo seguidas por Cuenca y Talavera de la Reina. Las que presentan una mayor calidad media de sus publicaciones medida por el factor de impacto de la revista de publicación son las de Guadalajara y Talavera de la Reina (Factor de Impacto medio en todas las áreas: 1,52). Los artículos que despiertan mayor interés en la comunidad profesional y científica son las de Puertollano, Ciudad Real y Talavera (número medio de citas por artículo en el conjunto de las áreas: 4,38). Las áreas cuyos artículos no reciben ninguna cita con mayor frecuencia son Guadalajara, Toledo y Albacete.

En la tabla 1 se aprecian las revistas con mayor número de artículos publicados, destacando Atención Primaria, Semergen y Salud Rural. En total la produc- ción científica de Primaria se recoge en 104 revistas diferentes.

\section{¿QUÉ ESTRATEGIAS SERÍAN ADECUADAS PARA IMPULSAR LA INVESTIGACIÓN EN ATENCIÓN PRIMARIA EN CASTILLA-LA MANCHA?}

A la vista de los datos ofrecidos anteriormente, ¿cuál podría ser la conclusión sobre el estado de la investigación en Atención Primaria en Castilla-La Mancha? La situación muestra luces y sombras y podría haber muchas interpretaciones posibles. Probablemente no sea tan negativa como a veces pensamos, pero muestra algunos signos de cierto estancamiento en los últimos años.

Sea como fuere, es cierto que siempre se podrá y se deberá mejorar. Existen numerosos estudios en la bibliografía que han analizado las causas y las condiciones para mejorar la investigación en Atención Primaria $^{2-11}$. En ellos se han utilizado diversas metodologías que van desde el análisis de grupos de expertos a encuestas realizadas a profesionales. Baste como ejemplo la relación de posibilidades de mejora que se ofrece en la tabla 2 extraída de la cita $\mathrm{n}^{\circ} 2$ (http://www.fisterra.com). La mejora en cualquiera de estos aspectos sin duda contribuiría a aumentar la cantidad y calidad de la investigación.

Pero probablemente el factor determinante cuyo cambio podría significar un avance cualitativo fundamental es pasar de la situación actual, en que la investigación en Atención Primaria está basada en el voluntarismo del profesional, a una situación en que la investigación constituya una obligación para la organización. Esto supondría que la organización tendría que conseguir al cabo del año unos objetivos de I+D del mismo rango que los objetivos asistenciales. Para ello tendría que poner en marcha algunos cambios muy importantes:

1. Personal. La organización debería contar con personal de atención primaria dedicado a la investigación. Podrían ser profesionales de atención primaria muy bien seleccionados, con mayor motivación y experiencia, que dedicaran por ejemplo el $50 \%$ de su jornada laboral a la investigación, sin perder contacto con la asistencia ni desde luego ver mermadas sus retribuciones. También podrían algunos de ellos dedicarse a tiempo completo. No es necesario (ni seguramente posible) que todos los profesionales se dediquen a la investigación. Sí lo es que exista un número mínimo de profesionales en la región (idealmente en todas las áreas sanitarias) que constituyan la masa crítica necesaria y estable para que la investigación se consolide. En torno a ellos se generarían grupos de investigación y otros profesionales podrían aprender 
a investigar investigando. Estos investigadores serían evaluados periódicamente y en una situación ideal podría haber competencia entre ellos para obtener el puesto de investigador por tres o cinco años. Sin perjuicio de esto, se mantendría el resto de la investigación realizada, como en la actualidad, por profesionales puramente "asistenciales".

2. Unidades de apoyo a la investigación. Se trata de unidades de tipo administrativo encaminadas a facilitar las tareas no puramente científicas de los investigadores (información sobre convocatorias, seguimiento y gestión de las memorias de los proyectos, cumplimentación de documentos, etc.). Además, sería muy deseable que contaran con un profesional experto en metodología de la investigación para asesorar a todos los investigadores en aspectos metodológicos.

\section{BIBLIOGRAFÍA}

1. Segura-Fragoso A. La producción científica de Castilla-La Mancha en Biomedicina y Ciencias de la Salud, 1973-2003. Toledo: FISCAM; 2006.

2. Pita Fernández S, Pértega Díaz S. Dificultades de la investigación en Atención Primaria. http://www fisterra com/mbe/inves-
tiga/diflnvestAP/diflnvesAP asp 2005 April 30Available from: URL: http://www.fisterra.com/mbe/investiga/diflnvestAP/diflnvesAP.asp

3. Ovhed I, van RP, Hakansson A. What is the future of primary care research? Probably fairly bright, if we may believe the historical development. Scand J Prim Health Care 2005; 23(4):248-53.

4. De Maeseneer JM, van Driel ML, Green LA, van WC. The need for research in primary care. Lancet 2003; 362(9392):1314-9.

5. Balague M, Valderas JM, Bolibar B. Research opportunities and organizational aspects in Primary Health Care. Med Clin (Barc) 2007; 128(18):711-4.

6. Diogene E, Pujol J, Juncosa S. Primary health care research as source of knowledge. Med Clin (Barc) 2007; 128(16):634-7.

7. Cevallos GC, Garrido ES, Lopez OM, Cervera BE, Estirado GA. Primary Care research: attitudes and hindrances perceived by our doctors. Aten Primaria 2004; 34(10):520-5.

8. Fernandez FI. Research in primary care. Med Clin (Barc) 2005; 124(2):57-60.

9. Jimenez VJ. Commentary: we need more and better research in primary care. Aten Primaria 2004; 34(10):525-7.

10. Pedraza M, V. Research in primary health care. Basic premises. Aten Primaria 2004; 34(6):318-22.

11. Peiro S, Artells Herrero JJ. Management of research in healthcare centers. An exploration through nominal group dynamic]. Gac Sanit 2001; 15(3):245-50. 